\title{
Germanica
}

\section{Ich und die Anderen: Kemal Kayankaya auf dem Weg in die Bürgerlichkeit}

Moi et les autres: Kemal Kayankaya sur le chemin de l'embourgeoisement

Me and the others: Kemal Kayankaya on the way to the middle class

\section{Stefan Seeber}

\section{(2) OpenEdition}

\section{Journals}

Édition électronique

URL : http://journals.openedition.org/germanica/3217

DOI : $10.4000 /$ germanica.3217

ISSN : 2107-0784

Éditeur

Université de Lille

Édition imprimée

Date de publication : 30 septembre 2016

Pagination : 189-197

ISBN : 9782913857377

ISSN : 0984-2632

Référence électronique

Stefan Seeber, «Ich und die Anderen: Kemal Kayankaya auf dem Weg in die Bürgerlichkeit »,

Germanica [Online], 58 | 2016, Online erschienen am: 30 September 2018, abgerufen am 06 Oktober 2020. URL : http://journals.openedition.org/germanica/3217 ; DOI : https://doi.org/10.4000/ germanica.3217

(c) Tous droits réservés 


\section{Roman policier, migration et société}





\section{Ich und die Anderen: Kemal Kayankaya auf dem Weg in die Bürgerlichkeit}

Stefan SEEBER

Universität Freiburg

\section{Arjouni und der hard-boiled Kriminalroman}

Arjounis Frankfurter Privatermittler Kemal Kayankaya ist - auf den ersten Blick - ein hard-boiled detective alter Schule, geformt nach den Vorbildern Hammetts und Chandlers' ${ }^{1}$ : Als ein Außenseiter türkischer Herkunft, aber mit deutschem Pass ist er stereotypen Alltagsrassismen ausgesetz ${ }^{2}$, die dazu beitragen, ein düsteres Bild von der Großstadt zu zeichnen, in der er ermittelt ${ }^{3}$, und die ihm trotzdem als Heimat ans

1. - Vgl. zur Skizze der Gattung Ulrich Suerbaum, Krimi. Analyse einer Gattung, Stuttgart, Reclam, 1984, S. 127-160 und Peter Nusser, Der Kriminalroman, 4. aktualisierte und erweiterte Auflage, Stuttgart/Weimar, Metzler, 2009 (SM 191), S. 124-136. Zu Arjouni im Kontext der amerikanischen Tradition vgl. Arlene A. Teraoka, „Detecting Ethnicity: Jakob Arjouni and the Case of the Missing German Detective Novel“, in: The German Quarterly 72,3 (1999), S. 265-289, hier S. 265.

2. - Vgl. dazu Mahmut Karakus, ,Jakob Arjounis Roman Ein Mann, ein Mord. Ermittlung in doppelter Angelegenheit“, in: Jean-Marie Valentin/Stéphane Pesnel (Hgg.): Akten des XI. Internationalen Germanistenkongresses Paris 2005. „Germanistik im Konflikt der Kulturen “, Bern u.a., Lang, Bd. 6, S. 281-286.

3. - Zu Frankfurt als Un-Ort bei Arjouni vgl. auch Thomas Kniesche, ,Vom Modell Deutschland zum Bordell Deutschland. Jakob Arjounis Detektivromane als literarische Konstruktionen bundesrepublikanischer Wirklichkeit“, in: Sandro M. Moraldo (Hg.), 
Herz gewachsen ist (MM 69, K 37) ${ }^{4}$. Er arbeitet in einem Umfeld voller Gewalt und befindet sich privat wie dienstlich die meiste Zeit am Rande der Gesellschaft, vorzugsweise im Rotlicht-Milieu, das vor allem die ersten drei Krimis der Reihe thematisch prägt. Anders als die Ermittler der amerikanischen hard-boiled Romane ist Kayankaya dabei für seine Rezipienten allerdings keine stereotype und „fixe Größe“" sich. Das wird besonders in den späten Romanen deutlich, vor allem in Bruder Kemal (2012) mit seinem verbürgerlichten Helden ${ }^{6}$. Solche Züge des Arrivierens kombiniert Arjouni gezielt mit dem Weiterbestehen von Elementen des harten Ermittlers, der Kayankaya immer noch ist: Diese prismenhafte Brechung der Figur ironisiert den Helden ${ }^{7}$ und fügt der breiten Palette an Sinnebenen der Werke eine weitere Nuance hinzu: Denn bereits von Anfang an nutzt Arjouni seine Romane, durchaus auch unter Einsatz von Ironie und Komik, um Genregrenzen zu transzendieren und politisch bzw. gesellschaftskritisch zu arbeiten, so dass die Kayankaya-Reihe Plattform für die Diskussion der Ghettoisierung von Gastarbeitern (HB), der ökologischen Bewegung (MB), von Flüchtlingsleid und Zwangsprostitution (MM), dem Jugoslawienkrieg (K) sowie von radikalem Islam (BK) wird. Auf diese Weise schafft Arjouni einen Mehrwert, der den literarischen Anspruch seiner Romane ausmacht, und er gibt Kayankaya ein eigenes Profil.

\section{Kayankaya im sozialen Raum}

Mein Ziel ist es, im Folgenden nachzuzeichnen, wie Kayankayas Weg durch die Reihe der fünf Kriminalromane vom Ostend ins Westend Frankfurts führt und wie er den ,sozialen Raum“ durchmisst, der ihm gegeben ist. Die Serie weist dabei eine deutliche Dynamisierung in der Charakterzeichnung des Helden auf: Die 1985, 1987 und 1991 publizierte Trilogie zeichnet noch relativ statisch das Bild des Underdog

Mord als kreativer Prozess. Zum Kriminalroman in Deutschland, Österreich und der Schweiz, Heidelberg, Winter, 2005 ( Beiträge zur neueren Literaturgeschichte 222), S. 21-39, hier S. 21.

4. - Ich zitiere die Werke im fortlaufenden Text mit den Siglen HB für Happy Birthday, Türke! (1985, ${ }^{2}$ 1987), MB für Mehr Bier (1987), MM für Ein Mann, ein Mord (1991), K für Kismet (2001) und BK für Bruder Kemal (2012). Alle Romane sind bei Diogenes in Zürich erschienen, nur die Erstauflage von HB erschien im Buntbuch-Verlag.

5. - Suerbaum a.a.O., S. 143.

6. - Gerrit Bartels, „Kriminalroman: Bruder Kemal. Von Borste zu Bratwurst“, in: Der Tagesspiegel, 24.01.2013, vgl. http://www.tagesspiegel.de/kultur/kriminalromanbruder-kemal-von-borste-zu-bratwurst/7677794.html spricht von „Verbürgerlichung“.

7. - Über Ironie und Komik in Arjounis Krimi-Reihe habe ich an anderem Ort Überlegungen angestellt, vgl. Stefan Seeber, „Allein unter Hessen. Formen der Komik in den Kayankaya-Romanen Jakob Arjounis“, in: Sabine Müller et al. (Hgg.): Mord und Totlach, Bochum/Freiburg,projektverlag, 2014 (Komik und Gewalt 6), S. 81-92. 
und präsentiert sich kompositorisch als geschlossene Einheit. Der erste Nachtrag, Kismet, 2001 erschienen, zeigt den alternden Helden und baut die Brücke zum bürgerlichen Ermittler in Bruder Kemal, der 2012 sein volles Potential der Verbürgerlichung entfaltet, vor allem aber seinen privaten Raum (Wohnung im Westend, der gehobenen Frankfurter Wohngegend) vom Arbeitsraum (im Ostend, der schlechten Gegend) trennt. Es lohnt sich deshalb, diese Zweiteilung des Werkes auch in der Analyse zu berücksichtigen und die Reihe nicht als ununterbrochenes Sinnkontinuum zu lesen.

Mit dem „sozialen Raum“ ist die Theorie Pierre Bourdieus aufgerufen, der in seinen Studien zu den Feinen Unterschieden ${ }^{8}$, aber auch in seiner Vorlesung Sozialer Raum und „Klassen"9 ein Panorama der zwischenmenschlichen Interaktion entwirft (er spricht vom „Raum von Beziehungen"10), in dem die unterschiedlichen Kapitalien (v. a. materiell, kulturell, sozial) in verschiedenen Kombinationen zu unterschiedlichen Habitus führen und damit den ,geheimnisvollen Abstimmungsmechanismus zwischen Gesellschaft und Individuum"11 steuern. Mit Dörner und Vogt ist, wenn Bourdieu für die Literatur nutzbar gemacht werden soll, deshalb danach zu fragen, welche sozialen Klassen im Text repräsentiert werden, welche Kapitalien sie mitbringen und wie diese Konstellation das „Machtfeld“ strukturieren, das den literarischen Raum prägt. ${ }^{12}$ Ich möchte noch einen Schritt weiter gehen und Bourdieus Vorstellung mit der Idee Goffmans von Statussymbolen und der gesellschaftlichen Stigmatisierung verbinden, die mir für Arjounis Romankonzeption sinnstiftend erscheint ${ }^{13}$. Goffman wird von Bourdieu oft aufgerufen, er setzt sich jedoch auch gezielt von ihm ab ${ }^{14}$, so dass ich damit einen Theoriensynkretismus betreibe, der z. T. konträre theo-

8. - Pierre Bourdieu, Die feinen Unterschiede. Kritik der gesellschaftlichen Urteilskraft, Frankfurt, Suhrkamp, 1987 (stw 658).

9. - Pierre Bourdieu, Sozialer Raum und „Klassen”. Leçon sur la leçon. Zwei Vorlesungen, übersetzt von Bernd Schwibs, mit einer Bibliographie der Schriften Pierre Bourdieus von Yvette Delsaut, Frankfurt, Suhrkamp, 1985 (stw 500).

10. - Bourdieu, Sozialer Raum, a.a.O., S. 13, im Original kursiv.

11. - Andreas Dörner/Ludgera Vogt, Literatursoziologie. Eine Einführung in zentrale Positionen - von Marx bis Bourdieu, von der Systemtheorie bis zu den British Cultural Studies, Heidelberg, Springer, 2., völlig überarbeitete und ergänzte Auflage 2013, S. 54.

12. - Dörner/Vogt, a.a.O., S. 57.

13. - Erving Goffman, Stigma. Über Techniken der Bewältigung beschädigter Identität, Frankfurt, Suhrkamp 1975 (stw140) und Ders., ,Symbols of Class Status“, in: The British Journal of Sociology 2 (1951), Heft 4, S. 294-304. Auf Goffman verweist auch Bourdieu, Sozialer Raum, a.a.O., S. 17 und, kritisch auf die Ausgrenzungsidee bezogen, Bourdieu, Die Feinen Unterschiede, a.a.O., S. 754.

14. - Vgl. dazu Boike Rehbein, Die Soziologie Pierre Bourdieus, Konstanz, UKV, 2006 (UTB 2778), S. 101f. 
retische Positionen im Dienste der Analyse kombiniert. Dabei geht es mir auch darum, das Kapital des Helden und seinen Status in Bezug auf die intendierten Rezipienten herauszuarbeiten, denen Arjouni grundlegende Bedeutung für die Sinnstiftung seiner Texte zumisst.

\section{Der junge Held als heldenhafter Außenseiter}

Der frühe Kayankaya ist ein outcast 15 : seine Kapitalien sind marginal, weder ökonomisch noch kulturell vermag er, sich dominant zu positionieren, sozial ist er marginalisiert, was Arjouni bis ins Detail ausmalt und als Markenzeichen entwickelt. Sein Held ist türkischer Herkunft, spricht aber kein Türkisch, und irrt eher orientierungslos durch seine Erwerbsbiographie. Er ist Anfeindungen der GastarbeiterCommunity ebenso ausgesetzt wie dem Alltagsrassismus von Behörden und den Einheimischen, auf die er trifft - sogar als Fußball-Fan, der Mönchengladbach präferiert, ist er in Frankfurt isoliert (MB 10, MM 5). Kayankaya nimmt sich selbst als depraviert wahr und begegnet seinem Leben mit genretypischer Lakonie, einen Aufstieg strebt er nicht an. Die Welt, in der er ermittelt und gegen die er ankämpft, ist feindlich und macht keine Sinnangebote; Bürgerlichkeit ist entweder spießig (paradigmatisch gefasst in der Figur des pensionierten Kommissars Löff, HB 86-91) oder wird als Fassade entlarvt, hinter der noch größere Traurigkeit lauert als im Leben des Helden (v. a. in der Figur der Ehefrau von Kommissar Futt, HB 138-142), Institutionen und Ämter sind korrupt und bieten keinen Schutz ${ }^{16}$ (vgl. z. B. die Gewaltorgie auf dem Polizeirevier in MB 70-79). Dem Ermittler bleibt nur der Rückzug auf die eigene Ethik und das Gefühl, der guten Sache zu dienen (MB 202f.): Er allein ist der Maßstab seines Handelns ${ }^{17}$.

Damit erarbeitet Arjouni eine Identität seines Helden Kayankaya, die ihm trotz des Mangels an ökonomischem wie kulturellem Kapital (sein Hobby ist neben Fußball - typisch für die 1980er Jahre - auch Tennis, MM 62f. bietet die Nacherzählung eines Ballwechsels von McEnroe in Echtzeit) großes symbolisches Kapital verschafft. Das muss vor dem Hintergrund von Bourdieus Theorie paradox erscheinen, denn Anerkennung erfährt der Held nicht innerhalb der

15. - Michael Porsche, „Hard-Boiled à la Turk: Jakob Arjouni’s Private Eye Kemal Kayankaya“, in: Peter Freese/Michael Porsche, Popular Culture in the United States. Proceedings of the German-American Conference in Paderborn, 14-17 September 1993, Essen, Die Blaue Eule, 1994 (Arbeiten zur Amerikanistik 12), S. 161-173, S. 163 nennt Kayankaya einen „outsider“.

16. - Diese Konstellation ist typisch für den hard-boiled Roman, vgl. Suerbaum, a.a.O., S, 128f.

17. - Ebd., S. 129. 
Romanwelt ${ }^{18}$, sogar die Lösung der ihm anvertrauten Fälle führt hier nicht zu Distinktionsgewinn, sondern affirmiert nur die lakonische Selbstbescheidung des einsamen Detektivs. Vielmehr wirkt Kayankaya nach außen, und Arjouni inszeniert durch seine Figurenzeichnung einen Pakt mit den Rezipienten, die mit dem Genre vertraut sind ${ }^{19}$, das er in seinen Romanen transzendiert. Der Held operiert vor dem Hintergrund der etablierten Gattung und variiert bekannte Muster, zu denen er sich neu positioniert: Der Einzelkämpfer-Detektiv erhält bei Arjouni menschliche Züge und wird verletzlich, das zeichnet ihn aus und verschafft ihm eine Respektposition. Zugleich werden die Attribute des Ermittlers als Statussymbole etabliert: Der Alkoholund Nikotinabusus, die Nachlässigkeit im Umgang mit Kleidung und Ernährung, die Verwahrlosung von Wohnung, Büro und Auto weisen Kayankaya als Angehörigen einer niedrigen Klasse aus, werden aber zugleich positiv als „Prestige-Symbole“ im Sinne Goffmans gesetzt, um ihn als Detektiv herauszuheben: Die Stigmatisierung des Ermittlers im Rahmen der Geschichte, indem ihm jedes Kapital abgesprochen und jeder Status verweigert wird, erweist sich in der Rezipientenlenkung als Auszeichnung 20 - Kayankaya ist dadurch zugehörig zur Gruppe der „harten“ Detektive mit eigenem Ethos und eigenen Wertvorstellungen. Die etablierten Eliten sind korrumpiert und nicht vertrauenswürdig21, der Außenseiter wird zur maßgeblichen Instanz für den Rezipienten: Dass Kayankaya dabei von geringer detektivischer Kompetenz ist und seine Fälle eher durch Zufall und Intuition als durch analytische Brillanz zu einem erfolgreichen Ende führt ${ }^{22}$, verstärkt diese Setzung noch zusätzlich: Der Ermittler avanciert zum Antihelden in einer feindseligen Welt, dem man gerade wegen seiner Unzulänglichkeit mit

18. - Bourdieu, Soziale Räume, a.a.O., S. 23.

19. - Zur hard-boiled-Begeisterung in der deutschsprachigen Krimi-Community der 1980er Jahre vgl. Suerbaum, a.a.O., S. 130.

20. - Zur Terminologie vgl. Goffman, Stigma, a.a.O., S. 58f.

21. - Zum negativen Bild vom Bürgertum vgl. Kniesche, a.a.O., S. 26, der allerdings die den Beschreibungen inhärente Komik völlig ausblendet und eine politische Homogenität der BRD voraussetzt, gegen die Arjouni anschreibe, die es aber so gar nicht gegeben hat.

22. - Es gibt zahlreiche Beispiele in allen Romanen der Serie, die die Begriffsstutzigkeit des Helden ebenso illustrieren wie seine Zufallstreffer, die ihn ans Ziel bringen. In HB z. B. verfolgt er - ohne dass es dafür irgendeinen Anhaltspunkt gäbe - über lange Zeit eine vermeintliche politische Spur und stellt nur beim Frühstück mit der Zeugin aufs Geratewohl die richtige Frage, die ihn weiterbringt (HB 77). Oft bringt er sich auch in Schwierigkeiten, die für die Lösung des Falles kontraproduktiv sind, etwa wenn er Zeugen gegen sich aufbringt oder die Polizei absichtlich auf sich aufmerksam macht (ebenfalls in HB Kommissar Futt, HB 33: „Ob es vernünftig war oder nicht, es machte Vergnügen $\left.[\ldots]^{\prime \prime}\right)$. 
Sympathie begegnet. Die Folie des amerikanischen hard-boiled Krimis dient dieser Figurenzeichnung als Hintergrund.

\section{Kayankaya auf dem Weg in die Bürgerlichkeit}

Erst nach zehn Jahren Pause ließ Arjouni der Trilogie einen weiteren Kayankaya-Roman folgen: Kismet spielt mit denselben Stereotypen (etwa mit Kayankaya unterwegs im Auftrag einer Orientalistin, die ihren Schäferhund[!] sucht, K 226-234), setzt stark auf das Mittel der Dialektkomik (K 119 u.ö.) und übersteigert die bekannte Brutalität ins apokalyptische Extrem eines Bandenkriegs mit Explosionen und Hinrichtungen ${ }^{23}$. Der Held hat an Gewicht zugelegt (K 5), ist weiterhin Raucher, chronisch unterfinanziert und einsam - die Figur Slibulski, eingeführt bereits in Mehr Bier als Helfer des Ermittlers, erhält hier als Familienersatz (K 28) und als einziger Freund Kayankayas noch deutlichere Konturen als in den vorangegangenen Texten. Alles Bürgerliche bleibt den Freunden fremd - Slibulskis Lebensgefährtin Gina, eine Archäologin, bildet mit ihrem akademischen Freundeskreis einen Kontrapunkt zum Lebensentwurf Kayankayas und wird umfangreich bloßgestellt (K 235-243). Der Held verweigert dich generell jeder Form der Verbürgerlichung: „Das Eckige, Niedrige, immer leicht nach irgendeinem Kleister oder Putzmittel Muffende gab mir ein Gefühl wie anderen der Geruch von Weihnachtsplätzchen" (K 184). Seine Beziehung zur Prostituierten Deborah (K 32) ist in der Krise und muss neu definiert werden (K 137), da sie auf einem Beschützerarrangement beruht, das seine Gültigkeit verloren hat: Der einsame Held steht an einem Scheideweg, der auch darin sinnbildlich gemacht wird, dass er in Kismet nicht mehr mit korrupten Eliten aneinander gerät (der korrumpierte, pädophile Kommissar Höttges wird von ihm als „Informationsquelle und direkter Hebel ins Polizeipräsidium“ ausgenutzt, da er den Polizisten mit Videomaterial erpresst [K 57], ist aber kein Gegner des Helden, nur Dienstleister). Kayankaya kämpft stattdessen zunächst als einzelner zwischen den zwei Banden, die den Frankfurter Untergrund dominieren, und er verbündet sich letztendlich mit Verbrechern: Der weiter aufrecht erhaltenen persönlichen Ethik (K 108) steht dieser Pakt entgegen, Kayankaya muss moralische Kompromisse schließen.

Diese Krise des Helden ist vor allem eine Krise der Rezeption des Helden: Der Detektiv hat seinen eindeutigen Ort eingebüßt und erscheint als Figur widersprüchlich und fragil. Nicht nur betrügt er Deborah mit einem namenlosen „Fräulein Kaugummi“ (K 265); ein Großteil des

23. - Bereits beginnend mit der Einstiegsszene, die in ihrer Figurenzeichnung parodistisch auf Quentin Tarantinos Pulp Fiction (1994) Bezug zu nehmen scheint (K 5-25). 
Romans wird darauf verwendet, seine Verliebtheit in die Mutter seiner Klientin Leila zu schildern und ihn erkennen zu lassen, dass er diese Frau, die als Mann verkleidet Schutzgelder für eine der verfeindeten Banden einzutreiben hatte, erschossen hat. Kayankaya hält die Fassade des lakonischen Ermittlers aufrecht, doch wird hier, anders als in den drei vorangegangenen Romanen, nicht der einsame, gerechte Held zelebriert und als Gegenentwurf zur korrumpierten Gesellschaft aufgebaut. Stattdessen wird den Rezipienten ein korrumpierter Held vorgeführt, der sich in Schuld verstrickt und der sich hinterfragt: ,[...] alle paar Wochen denke ich, wie schön es wäre, einen Garten oder einen Hof mit einer Wäscheleine zu haben“ (K 25). Der Anspruch: „Ich mußte dem Ganzen einen Sinn geben“ (K 35) wird im Roman gerade nicht eingelöst und katapultiert Kayankaya aus den zuvor gültigen Mustern und Strukturen heraus: Er hat weiterhin keinerlei Kapitalien, verliert jedoch sowohl seine Statussymbole (bildhaft gefasst im explodierten Büro [K 176f.]) als auch sein soziales Kapital, das auf der Stereotypie der Figur aufgebaut ist, die die Rezipienten im bekannten Schema zu verorten vermögen. Kayankaya ist in Bewegung geraten, doch bietet Kismet noch kein Ergebnis dieser Metamorphose.

Dieses Ergebnis erst wird im letzten Roman der Reihe, Bruder Kemal, entfaltet. Kayankaya gibt die Statusattribute des Detektivs auf: Er trinkt wesentlich weniger, wird zum Wein-Connaisseur (BK 90) und Espresso-Kenner (BK 127), er hat sich das Rauchen abgewöhnt (BK 32) und benutzt sein topisch schlecht gelegenes und heruntergekommenes Büro in Bahnhofsnähe (BK 35) im digitalen Zeitalter nur noch in Ausnahmefällen (BK 36), die eigentliche Arbeit erledigt er von zu Hause aus. Dieses Zuhause hält er geheim, um seine Freundin und deren Familie zu schützen, und auch die Rezipienten erfahren nur, dass es sich um „Vier-Zimmer-Küche-Bad im Westend“ (BK 37) handelt, also der gehobenen Wohngegend des Bürgertums. Seine Freundin hat die Prostitution aufgegeben und ein Lokal namens „Deborahs Naturweinstube“ (BK 90) eröffnet, um ihren Lebensunterhalt zu verdienen. Inszeniert wird auch ein Beinahe-Rückfall in die alten Gewohnheiten, der Kayankaya in eine Rotlichtkneipe führt, dort trinkt er zwei Bier und verlässt das Lokal „,erleichtert. Zu Hause aß ich einen Apfel, schaute „Tagesthemen“ und wartete auf Deborah" (BK 224). Den Roman beschließt die Nachricht seiner Lebensgefährtin, dass sie ein Kind erwarte, mit dem Kuss des Paares endet die Geschichte (BK 225) ${ }^{24}$.

Stärker als in den anderen Texten sind Privatleben und Ermittlung des Detektivs miteinander verwoben: Erstmals hat Kayankaya etwas zu

24. - Ob Arjouni damit eine geplante Fortsetzung der Geschichte Kayankayas insinuiert, muss offen bleiben, er ist kurz nach Erscheinen des Buches am 17. Januar 2013 gestorben. 
verlieren, und die hard-boiled Elemente (par excellence verwirklicht etwa in BK 200) werden kombiniert mit Fürsorge für seine Familie. Der outcast ist ein Bürger geworden: Wenn Habitus die „dauerhafte Disposition der Akteure" 25 bezeichnet, so führt uns Arjouni den Wandlungsprozess vor Augen, der mit dem Aufstieg im sozialen Raum verknüpft ist: Kayankaya durchläuft einen Geschmackswechsel weg vom Außenseiter hin zu einem mittleren Geschmack, die Rezipienten verfolgen einen Versuch des Distinktionsgewinns durch Akkumulation der Kapitalien, plakativ vorgeführt im Wohnortwechsel vom Ostend ins Westend. Entscheidend ist, und das macht $\mathrm{m}$. E. die Anwendung von Bourdieus Theorie auf Arjounis Helden besonders fruchtbar, dass der Wechsel nicht vollkommen gelingt, dass also die Herkunft des Detektivs nicht auszulöschen ist: „Dem Aufsteiger sieht man die Kletterei an" 26 und es vermengen sich Elemente der unterschiedlichen Geschmacksprägungen zu einem hybriden Gesamt. Der Habitus als „Erzeugungsprinzip objektiv klassifizierbarer Formen von Praxis und Klassifikationssystem (principium divisionis) dieser Formen" 27 wird dabei im Prozess des Entstehens vorgestellt, die Felder, in denen Kayankaya sich bewegt, überschneiden $\operatorname{sich}^{28}$.

Der Begriff des Hybriden weist darauf hin, dass hier eine neue Qualität des Erzählens des Helden über den Helden erreicht wird, denn Kayankaya berichtet als Ich-Erzähler über seine Metamorphose. Sie wird deshalb nicht reflektiert zugänglich gemacht, sondern muss aus den Hinweisen, die der Erzähler über sein Leben gibt, decodiert werden. Die Folgen der Veränderung sind weit reichend: An die Stelle der reihentypischen Lakonie am Ende der Geschichte treten Schwangerschaftsnachricht und Kuss, der hartgesottene Detektiv ist ,,alt geworden [...] und weich, und das ist gut so“(BK 189), der Rahmen der hard-boiled Kriminalgeschichte wird aufgebrochen und ergänzt durch eine biographische Entwicklung, die auch die Schwarzweißmalerei im Gesellschaftsbild relativiert: Die reiche, bürgerliche Auftraggeberin Kayankayas entpuppt sich als ehemalige „Fixernutte [...]“ (BK 223); der zweite Klient, ein muslimischer Schriftsteller, wird als Angeber und Hohlkopf bloßgestellt, mit ihm wird en passant der Betrieb der Frankfurter Buchmesse aufs Korn genommen. So wie die Stellung des Helden in der Gesellschaft ist die gesamte Gesellschaft in Fluss geraten und hat noch keine neue, greifbare Form gefunden - die Kapitalien

25. - Dörner/Vogt, a.a.O., S. 54.

26. - Bourdieu, „Soziale Räume“, a.a.O., S. 13. Kursiv im Original.

27. - Bourdieu, Die feinen Unterschiede, a.a.O., S. 277. Kursiv im Original.

28. - Vgl. dazu Sophia Prinz, ,Artikel Geschmack (goût)“, in: Gerhard Fröhlich/ Boike Rehbein, Bourdieu-Handbuch. Leben - Werk - Wirkung, Stuttgart/Weimar, Metzler, 2009, S. 104-110, hier S. 107. 
verändern ihre Wertigkeit, Habitus werden hinterfragbar, soziale Felder werden relativ und müssen neu generiert werden.

Das verändert zugleich den Anspruch an die Rezipienten, die nicht mehr einfach einen hard-boiled Detektiv vor der Folie eines genretypischen Umfeldes agieren sehen, sondern den Wandel der Figur nachvollziehen. An die Stelle der politischen Grundsatzdebatten besonders der ersten Romane tritt deshalb auch eine Detailbetrachtung von Einzelproblemen mit z. T. zynischem Unterton. Gesellschaftskritik wird privatisiert, der Ermittler wird neu aufgestellt, er funktioniert unabhängig vom Genremodell und wird stärker als Individuum wahrgenommen, das sich in der Welt zu verorten versucht: Im Zentrum steht die Frage nach dem ,sense of one's place“ 29 , nach der neuen Habitualisierung und den Schwierigkeiten, die dabei zu überwinden sind - der Rezipient sieht dem Helden dabei zu, wie er sich in einem Milieu als Basis für seinen neuen Habitus zu verankern versucht. Der stigmatisierte Außenseiter orientiert sich vor Publikum neu und beweist Aufstiegswillen.

Mit der Entwicklung des Helden bricht Arjouni das Schema des Kriminalromans auf - die Kapitalien werden in ihrer Gewichtung neu verhandelt, an die Stelle des Schemas tritt die Differenzierung: Dabei geraten beide Seiten, Held und Gesellschaft, aus dem Gleichgewicht, und kollektive wie individuelle Praktiken des Habitus ${ }^{30}$ werden fragil. Arjouni baut dabei auf die bereits in den ersten drei Bänden der Reihe erkennbare Anlage des Helden auf und nutzt den Mehrwert der ambiguen Figurenkonzeption eines Ermittlers, die er ausbaut und gerade auch im Hinblick auf den fragilen Habitus in einem unsicheren sozialen Raum problematisiert. Kayankaya ist immer ein Wiedergänger von Marlowe \& Co., er ist aber gleichzeitig auch immer wesentlich mehr - im Spannungsfeld von Selbst- und Fremdwahrnehmung, in den Überschneidungen von Gosse und Bürgerlichkeit und nicht zuletzt als Aufsteiger, dem man das Klettern deutlich ansieht.

29. - So zitiert Bourdieu, „Soziale Räume“, a.a.O., S. 17 Goffmann. Vgl. auch Pierre Bourdieu, Sozialer Sinn. Kritik der theoretischen Vernunft. Übersetzt von Günther Seib, Frankfurt, Suhrkamp, 1987, 114 zur Wahl des Milieus als Schutz des Habitus.

30. - Vgl. dazu Bourdieu, Sozialer Sinn, a.a.O., S. 101. 
